Published by Cambridge University Press.

This is an Open Access article, distributed under the terms of the Creative Commons Attribution licence (http://creativecommons.org/licenses/by/4.0/), which permits unrestricted re-use, distribution, and reproduction in any medium, provided the original work is properly cited. doi:10.1017/S1474748019000392

\title{
CHARACTERIZING O-MINIMAL GROUPS IN TAME EXPANSIONS OF O-MINIMAL STRUCTURES
}

\author{
PANTELIS E. ELEFTHERIOU ${ }^{\mathbb{1}}$ \\ Department of Mathematics and Statistics, University of Konstanz, Box 216, \\ 78457 Konstanz, Germany (panteleimon.eleftheriou@uni-konstanz.de) \\ (Received 3 September 2018; revised 15 June 2019; accepted 17 June 2019; \\ first published online 16 July 2019)
}

\begin{abstract}
We establish the first global results for groups definable in tame expansions of o-minimal structures. Let $\mathcal{N}$ be an expansion of an o-minimal structure $\mathcal{M}$ that admits a good dimension theory. The setting includes dense pairs of o-minimal structures, expansions of $\mathcal{M}$ by a Mann group, or by a subgroup of an elliptic curve, or a dense independent set. We prove: (1) a Weil's group chunk theorem that guarantees a definable group with an o-minimal group chunk is o-minimal, (2) a full characterization of those definable groups that are o-minimal as those groups that have maximal dimension; namely, their dimension equals the dimension of their topological closure, (3) as an application, if $\mathcal{N}$ expands $\mathcal{M}$ by a dense independent set, then every definable group is o-minimal.
\end{abstract}

Keywords: o-minimal structure; tame expansion; dimension function; definable group; independent set; group chunk

2010 Mathematics subject classification: Primary 03C64; 03C68

Secondary 22B99

\section{Introduction}

Definable groups have been at the core of model theory for at least a period of three decades, largely because of their prominent role in important applications of the subject, such as Hrushovski's proof of the function field Mordell-Lang conjecture in all characteristics [23]. Examples include algebraic groups (which are definable in algebraically closed fields) and real Lie groups (which are definable in o-minimal structures). Groups definable in o-minimal structures are well-understood, with arguably the most influential work in the area being the solution of Pillay's conjecture over a field [24], which brought to light new tools in theories with (Not the Independent Property) NIP. On the other hand, groups definable in tame expansions of o-minimal structures have only been studied locally [17]. In this paper we prove the first global results, whose gist is that one can recover an o-minimal group from an arbitrary definable group using only dimension-theoretic data.

The research was supported by an Independent Research Grant from the German Research Foundation (DFG) and a Zukunftskolleg Research Fellowship. 
O-minimal structures were introduced and first studied by van den Dries [9] and Knight-Pillay-Steinhorn [26, 32] and have since provided a uniform framework to study real algebraic and analytic geometry. They have enjoyed a wide spectrum of applications reaching out even to number theory and Diophantine geometry (such as in Pila's solution of certain cases of the André-Oort Conjecture [30]). In the meantime, several authors have sought more general structures that escape the o-minimal, locally finite setting, yet preserve the tame geometric behavior on the class of all definable sets. Such structures have heuristically been called 'tame'. An important category consists of those expansions $\mathcal{N}=\langle\mathcal{M}, \ldots\rangle$ of an o-minimal structure $\mathcal{M}$, where every open definable set (in $\mathcal{N}$ ) is already definable in $\mathcal{M}$. The primary example is the pair $\mathcal{N}=\langle\mathcal{M}, P\rangle$ of the real field $\mathcal{M}=\bar{R}$ expanded by the subfield $P$ of real algebraic numbers, studied by $\mathrm{A}$. Robinson in his classical paper [33], where the decidability of its theory was proven. Forty years later, van den Dries [10] extended Robinson's results to arbitrary dense pairs of o-minimal structures, and a stream of further developments in the subject followed $[2,3,6-8,14,22,27]$. Besides dense pairs, examples now include pairs of the form $\langle\mathcal{M}, P\rangle$, where $\mathcal{M}$ is an o-minimal expansion of an ordered group, and $P$ is a dense multiplicative subgroup with the Mann property, or a dense subgroup of the unit circle or of an elliptic curve, or it is a dense independent set.

Two indispensable tools in the study of definable sets and groups in the o-minimal setting have been the cell decomposition theorem and the o-minimal dimension [13]. In [17], the notion of a cell was extended to that of a 'cone' in a general setting that includes the above pairs $\mathcal{N}=\langle\mathcal{M}, P\rangle$, a relevant 'cone decomposition theorem' was established and an associated dimension function was studied. The setting of the current paper is even more general, as $\mathcal{N}=\langle\mathcal{M}, \ldots\rangle$ is an expansion of an o-minimal structure $\mathcal{M}$ where only the existence of a good dimension theory is assumed. Moreover, $\mathcal{M}$ does not need to expand an ordered group. Our main theorem (Theorem 1.1) strikingly reflects the idea that open definable sets are definable in $\mathcal{M}$, at the level of definable groups. Let us introduce some terminology and explain its concept.

Throughout this paper, $\mathcal{M}$ and $\mathcal{N}$ denote two first-order structures with the same universe $M$, and $\mathcal{N}$ expands $\mathcal{M}$. We denote by $\mathcal{L}$ the language of $\mathcal{M}$ and by del its usual definable closure. By ' $\mathcal{L}$-definable' or 'definable in $\mathcal{M}$ ' we mean definable in $\mathcal{M}$ with parameters. By 'definable' or 'definable in $\mathcal{N}$ ' we mean definable in $\mathcal{N}$ with parameters.

A dimension function compatible with $\mathcal{M}$ is a map $\operatorname{dim}$ from the class of all definable sets to $\{-\infty\} \cup \mathbb{N}$ that satisfies the following properties: for all definable $X, Y \subseteq M^{n}$, and $a \in M$,

(D1) $\operatorname{dim}\{a\}=0, \operatorname{dim} M=1$, and $\operatorname{dim} X=-\infty$ if and only if $X=\emptyset$,

(D2) $\operatorname{dim}(X \cup Y)=\max \{\operatorname{dim} X, \operatorname{dim} Y\}$,

(D3) if $\left\{X_{t}\right\}_{t \in I}$ is a disjoint definable family of sets, then

(a) for $d \in\{-\infty\} \cup \mathbb{N}$, the set $I_{d}=\left\{t \in I: \operatorname{dim} X_{t}=d\right\}$ is definable, and

(b) if every $X_{t}$ has dimension $k$, then

$$
\operatorname{dim} \bigcup_{t \in I} X_{t}=\operatorname{dim} I+k
$$


(D4) if $f: X \rightarrow Y$ is a definable bijection, then $\operatorname{dim} X=\operatorname{dim} Y$,

(D5) the dimension of every $\mathcal{L}$-definable set $X$ coincides with its usual o-minimal dimension,

(D6) every definable map $f: M^{n} \rightarrow M$ agrees with an $\mathcal{L}$-definable map $F: M^{n} \rightarrow M$ outside a definable set of dimension $<n$.

It follows from (D2) that dim is monotone, and from (D1)-(D4) that it is a dimension function also in the sense of [11].

In the rest of this paper, and unless stated otherwise, we assume that $\mathcal{N}$ admits a dimension function $\operatorname{dim}$ compatible with $\mathcal{M}$. With the exception of $\S 4, \mathcal{M}$ is o-minimal, and every open definable set is $\mathcal{L}$-definable.

In the aforementioned pairs $\langle\mathcal{M}, P\rangle$ the existence of such dim was established in [17] (details are postponed until $\S 2.4$ ). Recall from geometric stability theory that, given two sets $X \subseteq M^{n}$ and $Y \subseteq M^{m}, X$ is called $Y$-internal if there is a definable map $f: M^{m k} \rightarrow$ $M^{n}$ such that $X \subseteq f\left(Y^{k}\right)$. In the context of [17], it was shown there that a definable set $X$ has dimension 0 if and only if $X$ is internal to $P$ if and only if no open interval is internal to $X$. Such a set $X$ was called 'small'. In particular, $P$ is small. Likewise here, let us call a definable set small if it has dimension 0, and large, otherwise. Observe that by monotonicity of dim, the dimension of a definable set $X$ is bounded by the (o-minimal) dimension of its topological closure $c l(X)$. We call a definable set $X$ strongly large if

$$
\operatorname{dim} X=\operatorname{dim} c l(X) .
$$

We call a definable group strongly large if its domain is strongly large. Every infinite small set $X$ is not strongly large; indeed, $\operatorname{cl}(X)$ is $\mathcal{L}$-definable and infinite, and hence has positive dimension, whereas $\operatorname{dim} X=0$. On the other hand, every $\mathcal{L}$-definable set is strongly large. The main intuition is that, conversely, strongly large sets must behave like $\mathcal{L}$-definable sets. Our main theorem establishes this intuition at the level of definable groups.

Theorem 1.1. Let $\mathcal{N}$ be an expansion of an o-minimal structure $\mathcal{M}$ such that

(a) every open definable set is definable in $\mathcal{M}$, and

(b) $\mathcal{N}$ admits a dimension function compatible with $\mathcal{M}$.

Let $G$ be a definable group. Then $G$ is definably isomorphic to a group definable in $\mathcal{M}$ if and only if it is definably isomorphic to a strongly large group.

In the rest of this introduction, we discuss the content of the above theorem, state some consequences, and illustrate the main difficulties of its proof.

Theorem 1.1 is in the spirit of a classical theme in model theory; namely, to recover a mathematical object in some restricted language given data of possibly different nature. For example, the influential Pila-Wilkie theorem [31] recovers a semialgebraic subset of a set $X$ definable in an arbitrary o-minimal structure given a number-theoretic condition on $X$. In our case, we recover an o-minimal group from a group $G$ definable in an expansion of an o-minimal structure given a dimension-theoretic condition on the domain of $G$. 
We next point out the need for including definable isomorphisms in the conclusion of Theorem 1.1. Suppose $\mathcal{N}=\langle\overline{\mathbb{R}}, P\rangle$ is the expansion of the real field $\overline{\mathbb{R}}$ by the field $P$ of algebraic numbers. Let $K=\langle\mathbb{R},+\rangle$ and consider the definable bijection $f: P+$ $\pi P \rightarrow P^{2}$, with $f(x+\pi y)=(x, y)$. Let $G$ be the disjoint union of $K \backslash(P+\pi P)$ and $P^{2}$, equipped with the group structure induced from $K$ via the identity map on the first part and via $f$ on the second. Then $G$ is not strongly large, $\operatorname{since} \operatorname{dim} G=1$ and $\operatorname{dim} \operatorname{cl}(G)=2$, but it is definably isomorphic to the $\mathcal{L}$-definable group $K$.

Theorem 1.1 puts a constraint on the existence of new definable groups, which has already been the theme of previous research, such as in [4] and [5]. Let $\mathcal{N}=\langle\mathcal{M}, P\rangle$ denote one of the aforementioned pairs from [17]. As $P$ itself can be a definable group, there are new small definable groups (and a study for those has recently been initiated in [1]). Of course, there are also new large definable groups, such as the direct product $P \times M$, but as a consequence of the above theorem, there are no new large definable groups contained in $M$, or in any 1-dimensional $\mathcal{L}$-definable set. A special case of this statement was proven in [5, Lemma 7.3]; namely, that there are no new definable subgroups of 1-dimensional $\mathcal{L}$-definable groups. Returning to arbitrary dimensions, observe that, by (D3), $\operatorname{dim}(P \times M)=1$, whereas $\operatorname{dim} c l(P \times M)=M^{2}=2$. Hence $P \times M$ is not strongly large and Theorem 1.1 does not apply to it. What Theorem 1.1 implies is that there are no new definable groups of dimension $n$ contained in $M^{n}$, or in any $n$-dimensional $\mathcal{L}$-definable set.

In the example $\mathcal{N}=\langle\mathcal{M}, P\rangle$, where $P$ is a dense dcl-independent set, our work implies that there are no new definable groups at all. This pair recently received special attention in [8] and even triggered new model-theoretic work at the general level of ' $H$-structures' [4]. The basic intuition is that a dcl-independent set $P$ is at the other end of being a group, since there are no $\mathcal{L}$-definable relations between its elements. It is then easy to see that there are no new small definable groups, as those would have to be internal to $P$. Using the cone decomposition theorem from [17], we prove that every definable group is definably isomorphic to a strongly large group (Theorem 6.1). Combined with Theorem 1.1, we obtain the following application.

Theorem 1.2. Let $\mathcal{M}$ be an o-minimal expansion of an ordered group, and $\mathcal{N}=\langle\mathcal{M}, P\rangle$ an expansion of $\mathcal{M}$ by a dense dcl-independent set $P$. Then every group definable in $\mathcal{N}$ is definably isomorphic to a group definable in $\mathcal{M}$.

Again, a special case of this statement was previously proved, in [4, Proposition 6.4]; namely, that every definable subgroup of $\left\langle M^{n},+\right\rangle$ is $\mathcal{L}$-definable. As a parallel note, Theorem 1.2 applies also to interpretable groups, as those are definably isomorphic to definable ones (by elimination of imaginaries [8]). Elimination of imaginaries is known to fail in the general setting of Theorem 1.1 [7].

Finally, let us point out that Theorem 1.1 establishes a conjecture for definable groups stated in [15] and reformulated in [17], for the case of strongly large groups. Recall that a $\bigvee$-definable group is a group $\langle\mathcal{U}, \cdot\rangle$ whose universe is a directed union $\mathcal{U}=\bigcup_{k \in \mathbb{N}} X_{k}$ of definable subsets of $M^{n}$ for some fixed $n$, and for every $i, j \in \mathbb{N}$, the restriction of the group multiplication to $X_{i} \times X_{j}$ is definable. A map between two $\bigvee$-definable groups is called $\bigvee$-definable if its restriction to any definable subset of its domain is definable. 
The conjecture states that if $G$ is a definable group of dimension $k$, then there are a surjective group homomorphism $\tau: \mathcal{U} \rightarrow G$ and a short exact sequence

$$
0 \longrightarrow \mathcal{H} \longrightarrow \mathcal{U} \longrightarrow K \longrightarrow 0
$$

where $\mathcal{U}$ is $\bigvee$-definable, $\mathcal{H}$ is $\bigvee$-definable in $\mathcal{M}$ with $\operatorname{dim} \mathcal{H}=k, K$ is definable and small, and all maps involved are $\bigvee$-definable. Theorem 1.1 implies the conjecture for $G$ strongly large, with $\mathcal{U}=\mathcal{H}=G$ and $K$ trivial. Namely, it answers [17, Question 7.11] affirmatively. It is worth noting that the above conjecture was inspired by an analogous theorem in a different setting [19], namely that of semi-bounded o-minimal structures (see also Remark 4.1).

There are a number of different settings where at least the methods of this paper could apply, such as that of $H$-structures. Let us describe the strategy of our proof.

Sketch of the proof of Theorem 1.1. We illustrate the main difficulties in proving the right-to-left direction of Theorem 1.1. Given a strongly large group $G$, we need to recover a suitable $\mathcal{L}$-definable domain $X$ and an $\mathcal{L}$-definable map $F: X^{2} \rightarrow X$ that can play the role of an $\mathcal{L}$-definable group definably isomorphic to $G$. The construction is carried out in $\S 5$ through a series of six steps, which we list next. Let $G=\langle G, \cdot, 1\rangle$ be a strongly large group with $G \subseteq M^{n}$ and $\operatorname{dim} G=k$.

(I) Recover an $\mathcal{L}$-definable map $F: V^{2} \rightarrow M^{n}$, with $V \subseteq M^{n}$ and $\operatorname{dim}(G \triangle V)<k$, that agrees with . on a definable set $C \subseteq(V \cap G)^{2}$ with $\operatorname{dim}\left(V^{2} \backslash C\right)<2 k$. This is possible because $G$ is strongly large. Indeed, in Lemma 2.5 we generalize (D6) to maps $f$ with strongly large domain, such as $\cdot: G^{2} \rightarrow G$.

(II) Prove that $F$ satisfies 'group-like' properties on an $\mathcal{L}$-definable subset $U$ of $V^{2}$ with $\operatorname{dim}\left(V^{2} \backslash U\right)<2 k$, such as injectivity in each coordinate, and associativity. This is done using (I) and the fact that · is a group operation. Moreover, $F(U) \subseteq V$.

(III) Show that $F(U)$ contains an $\mathcal{L}$-definable set $X$ with $\operatorname{dim}(V \backslash X)<k$. This is done using earlier work from $\S 3$ for extracting $\mathcal{L}$-definable sets.

(IV) Construct a suitable definable embedding $h: X \rightarrow G$. This is the heart of the whole proof. We first prove that

(*) for every $t \in X$, there is unique $r \in G$, such that

the set $\{x \in V \cap G: F(t, x)=r \cdot x\}$ is of co-dimension $<k$ in $V$ (or $G$ ),

and then define $h(t)=r$ via $(*)$.

(V) Show that there is an $\mathcal{L}$-definable set $Z \subseteq X^{2}$ with $\operatorname{dim}\left(X^{2} \backslash Z\right)<2 k$ and $F(Z) \subseteq X$, such that for every $(t, x) \in Z$,

$$
h(F(t, x))=h(t) \cdot h(x) .
$$

The proof combines all information for $F, X$ and $h$ from Steps (II)-(IV).

(VI) Apply a group chunk theorem to the quadruple $(Z, X, F, h)$ to conclude that $G$ is definably isomorphic to an $\mathcal{L}$-definable group. This group chunk theorem is proved in $\S 4$ in a higher generality, where $\mathcal{M}$ and $\mathcal{N}$ are arbitrary structures satisfying only some of the dimension axioms (D1)-(D6). 
Example 1.3. We illustrate the above key step (IV) with one example. Suppose $\mathcal{N}=$ $\langle\mathcal{M}, P\rangle$ is an expansion of an ordered group $\mathcal{M}$ by a dense elementary substructure $P$. Let $K=\langle M,+\rangle$ and denote $\Gamma=M \backslash P$. Consider the definable bijection $h: M \rightarrow M$ given by

$$
h(x)= \begin{cases}x, & \text { if } x \in \Gamma, \\ x+1, & \text { if } x \in P\end{cases}
$$

and let $G=\langle M, \cdot\rangle$ be the induced group structure on $M$. Namely, for all $(t, x) \in M^{2}$,

$$
h(t+x)=h(t) \cdot h(x) .
$$

Then $G$ is strongly large (even with $\mathcal{L}$-definable domain), and it is definably isomorphic to the $\mathcal{L}$-definable group $K$ via $h$. We recover $h$ via $(*)$ above: we show that $h(x)$ is the unique element in $M$ such that the set $T=\{x \in M: t+x=h(x) \cdot x\}$ is co-small. Uniqueness is clear, since otherwise we would have two co-small disjoint subsets of $M$, a contradiction. To see that $T$ is co-small, consider the following equalities:

$$
t+x=h(t+x)=h(t) \cdot h(x)=h(t) \cdot x .
$$

The first equality holds for all those $x \in \Gamma$ such that $t+x \in \Gamma$, and hence, for co-small many $x$. The second equality holds for every $x \in M$. The third equality holds for all $x \in \Gamma$, again, co-small many. All together, $t+x=h(t) \cdot x$ holds for co-small many $x$.

Note that in this example, the rest of the ingredients of Steps (I)-(VI) above are: $V=M, C=\bigcup_{t \in \Gamma}\{t\} \times(\Gamma \cap(\Gamma-t)), F=+, U=V^{2}, X=F\left(\Gamma^{2}\right)=M$, and $U=X^{2}$.

Remark 1.4. The idea of recovering a group operation via $(*)$ is a recast of a similar idea in [28]. In $\S 1.3$ of that reference, the authors recover a definable isomorphism $h$ between $\langle M,<,+\rangle$ and an ordered group $\langle M,<, \oplus\rangle$, satisfying additional properties, as follows:

$$
h: t \mapsto \lim _{x \rightarrow \infty}[(t+x) \ominus x] .
$$

In Example 1.3, instead of letting $h(t)$ to be such a limit as $x \rightarrow \infty$, we require the equation $t+x=h(t) \cdot x$ to hold for co-small many $x$.

\section{Preliminaries}

In this section, we fix some notation, prove some basic facts, analyze strongly large sets, and show how the pairs $\langle\mathcal{M}, P\rangle$ from [17] fit into the current setting.

\subsection{Notation}

We assume familiarity with the usual notions of o-minimality, such as cells and cell decomposition (see, for example, [10]). By a $k$-cell, we mean a cell of dimension $k$. The topological closure of a set $X \subseteq M^{n}$ is denoted by $\operatorname{cl}(X)$. If $X, Z \subseteq M^{n}$, we call $X$ dense in $Z$ if $Z \subseteq \operatorname{cl}(X \cap Z)$. We call $X$ co-dense in $Z$ if $Z \backslash X$ is dense in $Z$. Given a set $X \subseteq M^{m} \times M^{n}$ and $a \in M^{m}$, we write $X_{a}$ for

$$
\left\{b \in M^{n}:(a, b) \in X\right\}
$$


unless stated otherwise. We write $\pi: M^{n} \rightarrow M^{n-1}$ for the projection onto the first $n$ coordinates, unless stated otherwise. If $X, Y \subseteq M$, we sometimes write $X Y$ for $X \cup Y$. A tuple of elements is denoted just by one element, and we write $b \subseteq B$ if $b$ is a tuple with coordinates from $B$. Our use of the notions of a open and closed box are standard. We say that a set $X$ has co-dimension $<k$ in $Y$, if $\operatorname{dim}(Y \backslash X)<k$. We write $X \triangle Y=(X \backslash Y) \cup$ $(Y \backslash X)$. We say that a map $f: A \subseteq M^{n} \times M^{n} \rightarrow M^{k}$ is injective in each coordinate if, for every $a, b \in M^{n}, f(a,-)$ and $f(-, b)$ are injective. We identify $M^{n} \times M^{n}$ with $M^{2 n}$. By an embedding we mean an injective map.

\subsection{Basic facts}

Fact 2.1. Let $V \subseteq M^{n}$ be a $k$-cell and $X \subseteq V$ a definable set with $\operatorname{dim}(V \backslash X)<k$. Then $X$ is dense in $V$.

Proof. Since $V$ is a $k$-cell, a relatively open subset $B$ of $V$ has dimension $k$. Since $\operatorname{dim}(B \backslash X)<k$, we obtain $\operatorname{dim}(B \cap X)=k$. In particular, $B \cap X \neq \emptyset$.

Fact 2.2. Let $Z \subseteq M^{n}$ be an $\mathcal{L}$-definable set of dimension $k$, and $Z^{\prime} \subseteq Z$ a $k$-cell. If a definable set is dense in $Z$, then it is dense in $Z^{\prime}$.

Proof. [16, Lemma 2.8].

The following lemma will be used in the proof of Lemma 5.4. It generalizes [17, Proposition 4.19].

Lemma 2.3. Let $F: X \subseteq M^{n} \rightarrow M^{m}$ be an $\mathcal{L}$-definable map, and $D \subseteq X$ a definable set with $\operatorname{dim}(X \backslash D)<\operatorname{dim} X$. Assume that $F_{\lceil D}$ is injective. Then there is an $\mathcal{L}$-definable set $Y \subseteq X$ such that $\operatorname{dim}(X \backslash Y)<\operatorname{dim} X$ and $F_{\lceil Y}$ is injective.

Proof. Assume $\operatorname{dim} X=k$. Denote

$$
T=\left\{a \in X: F^{-1}(F(a)) \text { is finite }\right\} .
$$

We claim that $\operatorname{dim}(X \backslash T)<k$. Assume not, and let $C \subseteq X \backslash T$ be a $k$-cell. Then $\operatorname{dim}(C \cap$ $D)=k$. Now, by [17, Fact 2.9], $F(C)$ has dimension $s<k$. In particular, $F(C)$ is in $\mathcal{L}$-definable bijection with a subset of $M^{s}$. Hence $C \cap D$ is in definable bijection with a subset of $M^{s}$, contradicting (D4).

Now, by uniform finiteness in o-minimal structures, one can easily find

- an $\mathcal{L}$-definable set $B \subseteq T$ of dimension $k$, such that $F_{\lceil B}$ is injective, and

- an $\mathcal{L}$-definable map $f: B \rightarrow T \backslash B$, such that for every $x \in B$,

$$
F(x)=F(f(x)) .
$$

Observe that then $f$ is injective, since if $x, y \in B$ and $f(x)=f(y)$, then $F(x)=$ $F(f(x))=F(f(y))=F(y)$ which implies $x=y$. Moreover, since $F_{\lceil D}$ is injective,

$$
f(B \cap D) \subseteq f(B) \backslash D .
$$

But $\operatorname{dim}(B \cap D)=k$, and hence by injectivity of $f$, the set on the right also has dimension $k$, contradicting $\operatorname{dim}(X \backslash D)<k$.

Question 2.4. In Lemma 2.3, can it moreover be $D \subseteq Y$ ? 


\subsection{Strongly large sets}

Here we prove some statements about strongly large sets. We also introduce the notion of a full set. The first lemma extends property (D6) to functions $f$ whose domain is any strongly large set, instead of just $M^{n}$.

Lemma 2.5. Let $X \subseteq M^{n}$ be strongly large of dimension $k$, and $f: X \rightarrow M^{m}$ a definable map. Then there are a definable set $S \subseteq X$ of dimension $<k$, and an $\mathcal{L}$-definable map $F: M^{n} \rightarrow M^{m}$, such that $f_{\lceil X \backslash S}=F_{\lceil X \backslash S}$.

Proof. By working with the coordinate functions of $f$, we may assume that $m=1$. Indeed, if we find a suitable set $S_{i}$ for the $i$-th coordinate $f_{i}$, then $S=\bigcup_{i} S_{i}$ works for $f$, by (D2).

We may assume $X \subseteq M^{k}$. To see this, observe that $c l(X)$ is a finite union of cells. If $C$ is one of the cells and $\operatorname{dim}(C \cap X)<k$, we can disregard it. Otherwise, $\operatorname{dim}(C \cap X)=k=$ $\operatorname{dim} \operatorname{cl}(C \cap X)$, and hence it is enough to work with one of these. After projecting $C$ onto suitable coordinates, we may assume that $X \subseteq M^{k}$.

Define $H: M^{k} \rightarrow M$ as $H(x)=f(x)$, if $x \in X$, and 0 , otherwise. This map $H$ is definable, and hence, by (D6), it agrees with an $\mathcal{L}$-definable map $F: M^{k} \rightarrow M$ outside a set $S$ of dimension $<k$. Then $f$ agrees with $F$ outside $S \cap X$.

The above lemma supports the intuition that strongly large sets behave like $\mathcal{L}$-definable sets. We strengthen the notion of being strongly large as follows.

Definition 2.6. A definable set $X$ is called full if $\operatorname{dim}(c l(X) \backslash X)<\operatorname{dim} X$.

By (D5), every $\mathcal{L}$-definable set is full. By (D2), a full set is strongly large. The converse is not true; for example, let $X$ be the disjoint union of an open interval and an infinite small set. Some natural examples of full sets come from the setting of [17], see Fact 2.12.

In $\S 5$, we will use the following consequence of (D6).

Corollary 2.7. Every strongly large set is a union of a full set and a set of smaller dimension.

Proof. Let $Y \subseteq M^{n}$ be a strongly large set of dimension $k$. As in the proof of Lemma 2.5, we may assume that $n=k$. Let $f: M^{n} \rightarrow M$ be a characteristic function for $Y$; namely, fix two elements $0,1 \in M$ and let $f(x)=1$, if $x \in Y$, and $f(x)=0$, otherwise. By (D6), $f$ agrees with an $\mathcal{L}$-definable map $F: M^{n} \rightarrow M$ outside a definable set $S$ of dimension $<n$. Let $C=\left\{x \in M^{n}: F(x)=1\right\}$. Since $f$ and $F$ agree on $C \backslash S$, this means that $C \backslash S \subseteq Y$. Since

$$
\operatorname{cl}(C \backslash S) \backslash(C \backslash S) \subseteq(\operatorname{cl}(C) \backslash C) \cup S,
$$

and the latter set has dimension $<n$, we obtain that $C \backslash S$ is full. Since also

$$
Y=(C \backslash S) \cup(Y \cap S),
$$

we are done.

The above conclusion may fail if we do not assume that the given set is strongly large. For example, consider any infinite small set. Also, in Corollary 6.8, we prove a partial 
converse of the above corollary for a pair $\mathcal{N}=\langle\mathcal{M}, P\rangle$ where $P$ is a dense dcl-independent set. We do not know whether that converse is true in general.

In $\S 6$, we will also need the following.

Lemma 2.8. A finite union of full sets is full.

Proof. Let $X=X_{1} \cup \cdots \cup X_{m}$, where each $X_{i}$ is a full set. Hence, $\operatorname{cl}(X)=\cup_{i} \operatorname{cl}\left(X_{i}\right)$, and

$$
c l(X) \backslash X=\left(\bigcup_{i} \operatorname{cl}\left(X_{i}\right)\right) \backslash\left(\bigcup_{i} X_{i}\right)=\bigcup_{i}\left(\operatorname{cl}\left(X_{i}\right) \bigcup_{i} X_{i}\right) \subseteq \bigcup_{i}\left(\operatorname{cl}\left(X_{i}\right) \backslash X_{i}\right),
$$

and hence $\operatorname{dim}(\operatorname{cl}(X) \backslash X) \leqslant \max _{i} \operatorname{dim}\left(\operatorname{cl}\left(X_{i}\right) \backslash X_{i}\right)<\operatorname{dim} X_{i} \leqslant \operatorname{dim} X$.

\subsection{The setting of $[17]$}

In [17] we studied pairs $\mathcal{N}=\langle\mathcal{M}, P\rangle$, where $P \subseteq M^{n}$, such that certain tameness conditions hold. In $[17, \S 2.2]$, the conditions were verified for the following examples: (a) dense pairs, (b) expansions of the real field by a dense multiplicative subgroup with the Mann property, or by a dense subgroup of the unit circle or of an elliptic curve, (c) expansions by a dense independent set. For the rest of this section, we work in the setting of [17]. Since we do not make any specific use of its conditions, we do not recall them, and the reader may consider $\mathcal{N}=\langle M, P\rangle$ to be any of the above examples. What we need from [17] is the notion of suitable dimension introduced there, which we describe next.

Definition 2.9 [17]. A supercone $J \subseteq M^{k}, k \geqslant 0$, and its shell $\operatorname{sh}(J)$ are defined recursively as follows:

- $M^{0}=\{0\}$ is a supercone, and $\operatorname{sh}\left(M^{0}\right)=M^{0}$.

- A definable set $J \subseteq M^{n+1}$ is a supercone if $\pi(J) \subseteq M^{n}$ is a supercone and there are $\mathcal{L}$-definable continuous maps $h_{1}, h_{2}: \operatorname{sh}(\pi(J)) \rightarrow M \cup\{ \pm \infty\}$ with $h_{1}<h_{2}$, such that for every $a \in \pi(J), J_{a}$ is contained in $\left(h_{1}(a), h_{2}(a)\right)$ and it is co-small in it. We let $\operatorname{sh}(J)=\left(h_{1}, h_{2}\right)_{\operatorname{sh}(\pi(J))}$.

Note that, $\operatorname{sh}(J)$ is the unique open cell in $M^{k}$ such that $\operatorname{cl}(\operatorname{sh}(J))=\operatorname{cl}(J)$.

Definition 2.10 (Large dimension [17]). Let $X \subseteq M^{n}$ be definable. If $X \neq \emptyset$, the large dimension of $X$ is the maximum $k \in \mathbb{N}$ such that $X$ contains a set of the form $f(J)$, where $J \subseteq M^{k}$ is a supercone and $f: \operatorname{sh}(J) \rightarrow M^{n}$ is an $\mathcal{L}$-definable continuous injective map. The large dimension of the empty set is defined to be $-\infty$.

The large dimension was used in [17] to prove a cone decomposition theorem for all definable sets, in analogy with the cell decomposition theorem known for o-minimal structures. A consequence of this theorem was that the large dimension satisfies all properties (D1)-(D6) of the current paper. More precisely, these properties are established in [17, Corollaries 5.3 and 5.5, Theorem 5.7, and Lemma 6.11]. Moreover, a definable set has large dimension zero if and only if it is small. We will make use of the cone 
decomposition theorem in $\S 6$, and we delay it until then. For now, let us point out some basic facts, again to be used in $\S 6$, but can be stated under the setting of [17].

In what follows, dim denotes the large dimension from [17].

Fact 2.11. Assume $\mathcal{N}$ is $\kappa^{+}$-saturated, where $\kappa$ is the size of its language. Let $X \subseteq M^{k}$ be a definable set of dimension $k$, and $A_{0} \subseteq M$ a finite set. Then there is $t \in X$ which is dcl-independent over $A_{0} P$.

Proof. By [17, Theorem 5.7(1)], $X$ contains a supercone, and hence we may assume that $X$ is a supercone. Consider the operator scl that maps $A \subseteq M$ to $\operatorname{scl}(A)=\operatorname{dcl}(A P)$. By $[17, \S 6]$, scl defines a pregeometry and the corresponding scl-dimension for definable sets agrees with dim. It is then easy to see from the definition of supercones, by induction on $k$, that there is $t \in X$ which is scl-independent over $A_{0}$, and hence dcl-independent over $A_{0} P$, as required.

In the next fact, we draw a connection to the full sets from the last subsection.

Fact 2.12. let $f(J)$ be as in Definition 2.10. Namely, $J \subseteq M^{k}$ is a supercone and $f: \operatorname{sh}(J) \rightarrow M^{n}$ is an $\mathcal{L}$-definable continuous injective map. Then $f(J)$ is a full set.

Proof. We first note that $J$ is a full set, by [17, Corollary 4.28]. Now, let $V=\operatorname{sh}(J)$. Observe that

$$
c l(f(J)) \backslash f(J) \subseteq c l(f(V)) \backslash f(J)=(c l(f(V)) \backslash f(V)) \cup(f(V) \backslash f(J)) .
$$

Since $f(V)$ is $\mathcal{L}$-definable, the first part of the last union has dimension $<k$. Since also $f$ is continuous and injective, the set

$$
f(V) \backslash f(J) \subseteq f(V \backslash J)
$$

also has $\operatorname{dimension} \operatorname{dim}(V \backslash J) \leqslant \operatorname{dim}(\operatorname{cl}(J) \backslash J)<k$, as needed.

\section{Local $\mathcal{L}$-definability}

This section contains a key result (Corollary 3.8) which will be used in the proof of Lemma 5.7 in order to extract an $\mathcal{L}$-definable set from some given data. The first step in the proof of Corollary 3.8 is to extract a 'locally $\mathcal{L}$-definable' set. Then, by Lemma 3.4 , every locally $\mathcal{L}$-definable set is $\mathcal{L}$-definable (Lemma 3.4 ).

\subsection{Preliminaries on local $\mathcal{L}$-definability}

Definition 3.1. Let $V \subseteq M^{n}$ be a definable set, and $x \in V$. We call $V$ locally $\mathcal{L}$-definable at $x$ if there is an open box $B \subseteq M^{n}$ containing $x$ such that $B \cap V$ is $\mathcal{L}$-definable. We call $V$ locally $\mathcal{L}$-definable if it is locally $\mathcal{L}$-definable at every point.

The following fact follows easily from the definition.

Fact 3.2. Suppose $V \subseteq M^{n}$ is a $k$-cell and $X \subseteq V$ a definable set. Then $X$ is locally $\mathcal{L}$-definable if and only if for every $x \in X$, there is a $k$-cell $B \subseteq X$ containing $x$. 
Of course, an $\mathcal{L}$-definable set is locally $\mathcal{L}$-definable. We will also prove the converse.

Lemma 3.3. Suppose $V \subseteq M^{n}$ is locally $\mathcal{L}$-definable and $X \subseteq M^{n}$ is $\mathcal{L}$-definable. Then $V \cap X$ is locally $\mathcal{L}$-definable.

Proof. If $B \subseteq M^{n}$ is an open box and $B \cap V$ is $\mathcal{L}$-definable, then so is $B \cap V \cap X$.

Lemma 3.4. A locally $\mathcal{L}$-definable set is $\mathcal{L}$-definable.

Proof. Let $V \subseteq M^{n}$ be locally $\mathcal{L}$-definable and suppose that the closure $C=\operatorname{cl}(V)$ has dimension $k$. We work by induction on $k$. If $k=0$, then $V$ is finite and hence $\mathcal{L}$-definable. Suppose $k>0$. We first prove that

$$
\operatorname{dim} \operatorname{cl}(C \backslash V)<k
$$

If not, there is a $k$-cell $C^{\prime} \subseteq C$ in which $C \backslash V$ is dense. Since $V$ is dense in $C$, by Fact 2.2 it is also dense in $C^{\prime}$. In particular, there is $x \in C^{\prime} \cap V$. Since $C^{\prime} \cap V$ is locally $\mathcal{L}$-definable (Lemma 3.3), there is a $k$-cell $B \subseteq C^{\prime}$ containing $x$ such that $B \cap V$ is $\mathcal{L}$-definable. But then both $B \cap V$ and $B \cap(C \backslash V)$ are dense in $B$. That is, $B \cap V$ and $B$ are both $\mathcal{L}$-definable and the former is dense and co-dense in the latter. However, by o-minimality, if $X, Y$ are two $\mathcal{L}$-definable sets and $X$ is dense in $Y$, then $\operatorname{dim}(Y \backslash X)<\operatorname{dim} Y$, implying that $X$ cannot be co-dense in $Y$. A contradiction.

By Lemma 3.3, the set $\operatorname{cl}(C \backslash V) \cap V$ is locally $\mathcal{L}$-definable. Since its closure is contained in $\operatorname{cl}(C \backslash V)$, by inductive hypothesis we obtain that it is $\mathcal{L}$-definable. Since

$$
V \backslash \operatorname{cl}(C \backslash V)=C \backslash \operatorname{cl}(C \backslash V)
$$

is also $\mathcal{L}$-definable, we conclude that $V$ is $\mathcal{L}$-definable.

Although it will not be used in this paper, we note that local $\mathcal{L}$-definability at a point is a definable notion.

Lemma 3.5. Let $V \subseteq M^{n}$ be a definable set. Then the set $S$ of points in $V$ at which $V$ is locally $\mathcal{L}$-definable is $\mathcal{L}$-definable.

Proof. It is easy to see that for any point $x \in V$, we have that $V$ is locally $\mathcal{L}$-definable at $x$ if and only if there is a closed box $B \subseteq M^{n}$ containing $x$ such that $\operatorname{cl}(B \cap V)=B \cap V$. Therefore, $S$ is definable. By its definition, it is thus locally $\mathcal{L}$-definable. By Lemma 3.4 , it is $\mathcal{L}$-definable.

\subsection{Extracting local $\mathcal{L}$-definability}

A simple and illustrative example of what follows is this. Let $\Gamma$ be the set of non-algebraic real numbers, and $f: \mathbb{R}^{2} \rightarrow \mathbb{R}$ the usual addition. Then $f(\Gamma \times \Gamma)=\mathbb{R}$ is $\mathcal{L}$-definable. The statements that follow generalize this observation. The extra complication in proving Corollary 3.8 is due to the fact that the domain $U$ of the given $f$ is not a product of $\mathcal{L}$-definable sets, forcing us to first prove local $\mathcal{L}$-definability of $f\left(\Gamma^{2} \cap U\right)$, with the assistance of the preceding lemmas. 
Lemma 3.6. Let $S_{1}, S_{2} \subseteq M^{n}$ be two $k$-cells, and $\Gamma_{i} \subseteq S_{i}$ definable sets with $\operatorname{dim}\left(S_{i} \backslash \Gamma_{i}\right)$ $<k$, for $i=1,2$. Suppose $f: S_{1} \times S_{2} \rightarrow M^{k}$ is an $\mathcal{L}$-definable continuous map, injective in each coordinate, and let $x \in f\left(\Gamma_{1} \times \Gamma_{2}\right)$. Then $f\left(\Gamma_{1} \times \Gamma_{2}\right)$ contains an open subset of $M^{k}$ that contains $x$.

Proof. The proof is inspired by an example in [3, page 5]. Let $x=f(a, b)$, where $(a, b) \in \Gamma_{1} \times \Gamma_{2}$. We first claim that there is a definable set $\Gamma_{1}^{\prime} \subseteq \Gamma_{1}$ of dimension $k$, such that $\bigcap_{t \in \Gamma_{1}^{\prime}} f\left(t, S_{2}\right)$ contains an open set $K$ that contains $x$. Since $f(a,-): S_{2} \rightarrow M^{k}$ is $\mathcal{L}$-definable, continuous and injective, by [25] $f\left(a, S_{2}\right)$ contains an open set that contains $f(a, b)$. By continuity of $f$, there is a $k$-cell $I \subseteq S_{1}$ containing $a$, such that $\bigcap_{t \in I} f\left(t, S_{2}\right)$ contains an open set that contains $f(a, b)$. We can thus set $\Gamma_{1}^{\prime}=I \cap \Gamma_{1}$, which has dimension $k$.

Now let $\Gamma_{1}^{\prime}$ and $K$ be as above. We prove that actually $f\left(\Gamma_{1}^{\prime} \times \Gamma_{2}\right)$ contains $K$. Assume toward a contradiction that there is $m_{0} \in K$ such that $m_{0} \notin f\left(\Gamma_{1}^{\prime} \times \Gamma_{2}\right)$. By the claim in the first paragraph,

$$
m_{0} \in \bigcap_{t \in \Gamma_{1}^{\prime}} f\left(t, S_{2} \backslash \Gamma_{2}\right) .
$$

By injectivity of $f$ in the first coordinate, we obtain

$$
\Gamma_{1}^{\prime} \subseteq f\left(-, S_{2} \backslash \Gamma_{2}\right)^{-1}\left(m_{0}\right)
$$

which is a contradiction, because $\operatorname{dim}\left(S_{2} \backslash \Gamma_{2}\right)<k=\operatorname{dim}\left(\Gamma_{1}^{\prime}\right)$, and hence its image under the definable map $x \mapsto f(-, x)^{-1}\left(m_{0}\right)$ cannot contain $\Gamma_{1}^{\prime}$ (see, for example, $[17$, Corollary 5.3]).

We next derive a version of the last lemma where the range of $f$ is a $k$-cell in any $M^{n}$.

Lemma 3.7. Let $S_{1}, S_{2}, V \subseteq M^{n}$ be three $k$-cells, and $\Gamma_{i} \subseteq S_{i}$ definable sets with $\operatorname{dim}\left(S_{i} \backslash\right.$ $\left.\Gamma_{i}\right)<k$, for $i=1,2$. Suppose $f: S_{1} \times S_{2} \rightarrow V$ is an $\mathcal{L}$-definable continuous map, injective in each coordinate, and let $x \in f\left(\Gamma_{1} \times \Gamma_{2}\right)$. Then $f\left(\Gamma_{1} \times \Gamma_{2}\right)$ contains a $k$-cell that contains $x$.

Proof. Suppose $x=f(a, b)$, for $(a, b) \in \Gamma_{1} \times \Gamma_{2}$. Let $\pi: M^{n} \rightarrow M^{k}$ be a coordinate projection which is injective on $V$. Then $F:=\pi \circ f: S_{1} \times S_{2} \rightarrow M^{k}$ is an $\mathcal{L}$-definable continuous map that satisfies the conditions of Lemma 3.6. So $F\left(\Gamma_{1} \times \Gamma_{2}\right)$ contains an open box $K$ of $M^{k}$ that contains $\pi(x)$. Then $(\pi / V)^{-1}(K) \subseteq f\left(\Gamma_{1} \times \Gamma_{2}\right)$ is a $k$-cell that contains $x$.

It is not hard to see that the above lemma remains true if $V$ is any $\mathcal{L}$-definable set of dimension $k$, but we will not need this fact here. However, if $V$ is of higher dimension, then the lemma fails: let $f$ be the identity map and $\Gamma$ contain no open $\mathcal{L}$-definable set.

We can now prove the exact statement that will be used in the proof of Lemma 5.7.

Corollary 3.8. Let $V \subseteq M^{n}$ be a $k$-cell, $U \subseteq V^{2}$ a finite union of $2 k$-cells, and $\Gamma \subseteq V$ a definable set with $\operatorname{dim}(V \backslash \Gamma)<k$. Suppose that $f: U \rightarrow V$ is an $\mathcal{L}$-definable continuous map, which is injective in each coordinate. Then the set $X=f\left(\Gamma^{2} \cap U\right)$ is $\mathcal{L}$-definable. 
Proof. By Lemma 3.4, it suffices to show that $X$ is locally $\mathcal{L}$-definable. So let $x=F(a, b)$, where $(a, b) \in \Gamma^{2} \cap U$. Since $U \subseteq V^{2}$ is a finite union of $2 k$-cells and $\operatorname{dim} V=k$, it is easy to find $k$-cells $S_{1}, S_{2} \subseteq V \subseteq M^{n}$ such that $(a, b) \in S_{1} \times S_{2} \subseteq U$. Since $S_{i} \subseteq V$, we have $\operatorname{dim}\left(S_{i} \backslash \Gamma\right)<k$, and hence by Lemma 3.7, for $\Gamma_{i}=\Gamma \cap S_{i}$, there is a $k$-cell $B$ with

$$
x \in B \subseteq F\left(\left(\Gamma \cap S_{1}\right) \times\left(\Gamma \cap S_{2}\right)\right) \subseteq X .
$$

By Fact $3.2, X$ is locally $\mathcal{L}$-definable.

\section{A Weil's group chunk theorem}

The goal of this section is to recover an $\mathcal{L}$-definable group from an $\mathcal{L}$-definable 'group chunk'. Theorems of this spirit have already been considered in classical model theory. The current account borrows ideas from Weil's group chunk theorem as it appears in van den Dries [12]. The proof of Theorem 4.4 is based on discussions with Peterzil.

In this section, we work in a more general setting than in the rest of this paper. Let $\mathcal{M}$ and $\mathcal{N}$ be any two first-order structures, with $\mathcal{N}$ expanding $\mathcal{M}$. Assume that there is a map dim from the class of all definable sets in $\mathcal{N}$ to $\{-\infty\} \cup \mathbb{N}$, such that the following properties from the introduction hold:

- (D1), (D2), (D3b), (D4), and

- if the family $\left\{X_{t}\right\}_{t \in I}$ in (D3) is $\mathcal{L}$-definable, then so are the sets $I_{d}$ in (D3a).

We refer to the second property above as (Ldef). Note that we do not assume that $\mathcal{M}$ is o-minimal, nor that it admits a dimension function. But even with an o-minimal $\mathcal{M}$, the current setting is much richer than in the rest of the paper. For example, it includes $d$-minimal structures $[21]$, such as $\mathcal{N}=\left\langle\mathbb{R},<,+, \cdot, 2^{\mathbb{Z}}\right\rangle$, and also weakly o-minimal non-valuational structures [34], such as $\mathcal{N}=\langle\mathbb{Q},<,+,(0, \pi)\rangle$.

Remark 4.1. An example where $\mathcal{N}$ satisfies the above properties, but not (D3a), is that of a semi-bounded o-minimal structure $\mathcal{N}=\langle\mathcal{M}, P\rangle$; namely, when $\mathcal{M}$ is a linear o-minimal structure, $P$ is an o-minimal expansion of a real closed field defined on a bounded interval, and $\operatorname{dim}$ is the usual o-minimal dimension [29, Proposition 3.6]. Even though groups definable in semi-bounded o-minimal structures are already well-understood [19], the results of this section appear to be new also in that setting.

Under these assumptions, we recover a group which is interpretable in $\mathcal{M}$. This will be enough for our purposes in this paper, in view of Fact 4.3. To avoid any ambiguities, let us recall the following definition from [20].

Definition 4.2. Let $\mathcal{R}$ be any structure. By a definable quotient (in $\mathcal{R}$ ) we mean a quotient $X / E$ of a definable set $X$ by a definable equivalence relation $E$. A map $\tau: X / E_{1} \rightarrow Y / E_{2}$ between two definable quotients is called definable if the set

$$
\{(x, y) \in X \times Y: \tau([x])=[y]\}
$$

is definable (in $\mathcal{R}$ ). An interpretable group $G$ is a group whose universe is a definable quotient, and whose group operation is a definable map. 
Fact 4.3 [20, Theorem 1]. If $\mathcal{R}$ is o-minimal, then every interpretable group is definably isomorphic to a definable group.

We extend our terminology from the introduction to definable quotients: a quotient, map and group as in Definition 4.2, is called 'definable' or 'interpretable' if $\mathcal{R}=\mathcal{N}$, and ' $\mathcal{L}$-definable' or ' $\mathcal{L}$-interpretable' if $\mathcal{R}=\mathcal{M}$.

Theorem 4.4. Let $G=\langle G, \cdot, 1\rangle$ be a definable group with $G \subseteq M^{n}$ and $\operatorname{dim} G=k$. Suppose that

- $X \subseteq M^{m}$ and $Z \subseteq X^{2}$ are two $\mathcal{L}$-definable sets, with $\operatorname{dim}\left(X^{2} \backslash Z\right)<2 k$,

- $h: X \rightarrow G$ is a definable injective map, with $\operatorname{dim}(G \backslash h(X))<k$, and

- $F: X^{2} \rightarrow X$ is an $\mathcal{L}$-definable map, such that for every $(x, y) \in Z$,

$$
h(x) \cdot h(y)=h(F(x, y)) .
$$

Then $G$ is definably isomorphic to an $\mathcal{L}$-interpretable group. If, moreover, $\mathcal{M}$ is o-minimal, then $G$ is definably isomorphic to an $\mathcal{L}$-definable group.

Proof. We may assume that $X \subseteq G$ and $h=i d$. Indeed, one can form the disjoint union of $X$ and $G \backslash h(X)$, and induce on it a definable group structure after identifying $X$ with $h(X)$, and $G \backslash h(X)$ with itself. We then have $\operatorname{dim}(G \backslash X)<k$, $\operatorname{dim}\left(X^{2} \backslash Z\right)<2 k$, and $F: X^{2} \rightarrow X$ is an $\mathcal{L}$-definable map, such that for every $(x, y) \in Z$,

$$
x \cdot y=F(x, y) \text {. }
$$

To simplify the notation, for $a, b \in G$, we may write $a b$ for $a \cdot b$. Moreover, we may assume that for every $a \in X, \operatorname{dim}\left(X \backslash Z_{a}\right)<k$. Indeed, by (Ldef), the set $X^{\prime}=\{a \in$ $\left.X: \operatorname{dim}\left(X \backslash Z_{a}\right)<k\right\}$ is $\mathcal{L}$-definable. Moreover, since $\operatorname{dim}\left(X^{2} \backslash Z\right)<2 k$, it follows that $\operatorname{dim}\left(X \backslash X^{\prime}\right)<k$. We may thus replace $X$ by $X^{\prime}$. Finally, note that $F_{\uparrow Z}$ is injective in each coordinate.

Claim 1. The following sets are $\mathcal{L}$-definable:

$$
\begin{aligned}
& A=\left\{(a, b, c, d) \in X^{4}: a b=c d\right\}, \\
& B=\left\{(a, b) \in X^{2}: a b=1\right\} .
\end{aligned}
$$

Proof of Claim 1. We first prove that for every $a, b \in X$, the set

$$
T=\{x \in X: a b x=F(a, F(b, x))\}
$$

has co-dimension $<k$ in $X$. Indeed, every $x \in Z_{b}$ such that $F(b, x) \in Z_{a}$ is contained in $T$, by the third condition of the theorem. Now, $\operatorname{dim}\left(X \backslash Z_{b}\right)<k$, and by injectivity of $F_{\lceil Z}$ in the second coordinate, the set of $x \in X$ such that $F(b, x) \in Z_{a}$ also has co-dimension $<k$. It follows that $\operatorname{dim}(X \backslash T)<k$.

For $A$, we claim that for every $a, b, c, d \in X, a b=c d$ if and only if the set

$$
S=\{x \in X: F(a, F(b, x))=F(c, F(d, x))\}
$$

has co-dimension $<k$ in $X$. This will be enough, since the property on the right side of the above equivalence is $\mathcal{L}$-definable, by (Ldef). To see the above equivalence, assume first $a b=c d$. Then for every $x \in T$, we have

$$
F(a, F(b, x))=a b x=c d x=F(c, F(d, x)) .
$$


Since $T$ has co-dimension $<k$ in $X$, we are done. For the other direction, assume $S$ has co-dimension $<k$ in $X$. Then for every $x \in T \cap S$, we have

$$
a b x=F(a, F(b, x))=F(c, F(d, x))=c d x .
$$

Since both $S, T$ have co-dimension $<k$ in $X$, we have $T \cap S \neq \emptyset$, and hence there is $x \in X$ such that $a b x=c d x$. Therefore, $a b=c d$.

For $B$, first note that there are $c, d \in X$ with $c d=1$. Indeed, since $\operatorname{dim}(G \backslash X)<k$, we have $\operatorname{dim}\left(G \backslash X^{-1}\right)<k$, and hence $X \cap X^{-1} \neq \emptyset$. So we can take any $c \in X \cap X^{-1}$ and $d=c^{-1} \in X$. Now,

$$
B=\left\{(a, b) \in X^{2}:(a, b, c, d) \in A\right\},
$$

and hence it is $\mathcal{L}$-definable.

Let $\sim$ be the following equivalence relation on $X^{2}$ :

$$
(a, b) \sim(c, d) \Leftrightarrow a b=c d .
$$

By definability of the set $A$ from Claim 1 , the relation $\sim$ is $\mathcal{L}$-definable. Let $K=X^{2} / \sim$ and denote by $[(a, b)]$ the equivalence class of $(a, b)$. So $K$ is an $\mathcal{L}$-definable quotient. We aim to equip $K$ with an $\mathcal{L}$-interpretable group structure $\left\langle K, *, 1_{K}\right\rangle$.

Claim 2. (1) For every $a, b, c, d \in X$, there are $e, x, y, f \in X$, such that $a b=e x, c d=y f$ and $x y=1$.

(2) For every $a, b, c, d, s, t \in X$, there are $e, x, y, z, w, f \in X$, such that $a b=e x$, $c d=y z, s t=w f$ and $x y=z w=1$.

Proof of Claim 2. We only prove (1), as the proof of (2) is similar. Consider the sets

$$
S=\left\{(e, x) \in X^{2}: a b=e x\right\}
$$

and

$$
T=\left\{(y, f) \in X^{2}: c d=y f\right\} .
$$

Since $\operatorname{dim}(G \backslash X)<k$, the projections $\pi_{1}(T)$ and $\pi_{2}(S)$ on the first and last $m$ coordinates, respectively, have co-dimension $<k$ in $X$. In particular,

$$
\pi_{2}(S) \cap X \cap\left(\pi_{1}(T) \cap X\right)^{-1} \neq \varnothing .
$$

Now take $x$ in this set and let $y=x^{-1}, e=a b x^{-1}$ and $f=y^{-1} c d$. By construction, $x, y, e, f \in X$ and they satisfy the equalities of the conclusion.

Now, for every $a, b, c, d, e, f \in X$, define the relation

$$
R(a, b, c, d, e, f) \Leftrightarrow \text { there are } x, y \in X \text { such that } a b=e x, c d=y f \text { and } x y=1 .
$$

By Claim $1, R$ is an $\mathcal{L}$-definable relation. By Claim 2(1), for every $a, b, c, d \in X$, there are $e, f \in X$ such that $R(a, b, c, d, e, f)$. Moreover, if $R(a, b, c, d, e, f), R\left(a^{\prime}, b^{\prime}, c^{\prime}, d^{\prime}, e^{\prime}, f^{\prime}\right)$, $a b=a^{\prime} b^{\prime}$ and $c d=c^{\prime} d^{\prime}$, then $e f=e^{\prime} f^{\prime}$. Indeed, let $x, y, x^{\prime}, y^{\prime}$ witnessing the first two relations. Then

$$
e f=e x y f=a b c d=a^{\prime} b^{\prime} c^{\prime} d^{\prime}=e^{\prime} x^{\prime} y^{\prime} f^{\prime}=e^{\prime} f^{\prime} .
$$


We can thus define the following $\mathcal{L}$-definable operation on $K$ :

$$
[(a, b)] *[(c, d)]=[(e, f)] \Leftrightarrow R(a, b, x, d, e, f) .
$$

Let $1_{K}=[(x, y)]$ for some/any $x, y \in X$ such that $x y=1$. Namely, take $x \in X \cap X^{-1}$, which exists since $\operatorname{dim}(G \backslash X)<k$.

Claim 3. $K$ is an $\mathcal{L}$-interpretable group.

Proof of Claim 3. We already saw that the set $K$ and map $*$ are $\mathcal{L}$-definable. We prove associativity of $*$. Let $a, b, c, d, e, f \in X$. Take $e, x, y, z, w, f$ as in Claim 2(2). Then

$$
\begin{aligned}
([(a, b)] *[(c, d)]) *[(s, t)]=[(e, z)] *[(w, f)]=[(e, f)] & =[(e, x)] *[(y, f)] \\
& =[(a, b)] *([(c, d)] *[(s, t)]) .
\end{aligned}
$$

It is also easy to check that $1_{K}$ is the identity element, using Claim 2(1).

Claim 4. $K$ is definably isomorphic to $G$.

Proof of Claim 4. Let $\tau: K \rightarrow G$ be given by $[(a, b)] \mapsto a b$. By definition of $\sim, \tau$ is injective. It is also onto $\operatorname{since} \operatorname{dim}(G \backslash X)<k$ and hence for every $x \in G$, we can choose $a \in X \cap x X^{-1}$ and $b=a^{-1} x$. It remains to see that $\tau$ is a group homomorphism. Let $a, b, c, d \in X$, and take $e, x, y, f \in X$ as in Claim 2(1). We then have

$$
\tau([(a, b)] *[(c, d)])=\tau([(e, f)])=e f=\operatorname{exy} f=a b c d=\tau([(a, b)]) \cdot \tau([(c, d)]),
$$

as required.

The 'moreover' clause is clear by the definitions and Fact 4.3.

\section{The proof of Theorem 1.1 .}

We are now ready to prove Theorem 1.1. The left-to-right direction is immediate, since every $\mathcal{L}$-definable group is strongly large. For the right-to-left direction, we prove that any strongly large group satisfies the assumptions of Theorem 4.4.

Theorem 5.1. Let $G=\langle G, \cdot, 1\rangle$ be a strongly large group with $G \subseteq M^{n}$ and $\operatorname{dim} G=k$. Then there are

- $\mathcal{L}$-definable sets $X \subseteq M^{m}$ and $Z \subseteq X^{2}$, with $\operatorname{dim}\left(X^{2} \backslash Z\right)<2 k$,

- a definable injective map $h: X \rightarrow G$, with $\operatorname{dim}(G \backslash h(X))<k$, and

- an $\mathcal{L}$-definable map $F: X^{2} \rightarrow X$, such that for every $(x, y) \in Z$,

$$
h(x) \cdot h(y)=h(F(x, y)) .
$$

The rest of this section is devoted to proving Theorem 5.1. The proof runs through the five first steps mentioned in the introduction. For $a, b \in G$, we write $a b$ for $a \cdot b$.

\section{Step I : Recovering an $\mathcal{L}$-definable map $F$ from.}

The results on strongly large sets from $\S 2.3$ are used here. Note that in the next lemma, if the set $C$ were $\mathcal{L}$-definable, we would have already proved Theorem 5.1 (with $X=V$, $Z=C$, and $h=i d)$. 
Lemma 5.2. There are a closed $\mathcal{L}$-definable set $V \subseteq M^{n}$, with $\operatorname{dim}(G \triangle V)<k$, a definable set $C \subseteq(V \cap G)^{2}$, with $\operatorname{dim}\left(V^{2} \backslash C\right)<2 k$, and an $\mathcal{L}$-definable map $F: V^{2} \rightarrow M^{n}$, such that

$$
F_{\uparrow C}=\cdot \uparrow C
$$

Moreover,

$$
C=\bigcup_{t \in \Gamma}\{t\} \times C_{t},
$$

for some $\Gamma, C_{t} \subseteq V$, for $t \in \Gamma$, with $\operatorname{dim}(V \backslash \Gamma)<k$ and $\operatorname{dim}\left(V \backslash C_{t}\right)<k$.

Proof. By Corollary 2.7, $G$ is the union of a full set $G_{1}$ and a set of dimension $<k$. Let $V=\operatorname{cl}\left(G_{1}\right)$. Since $G_{1}$ is full, $\operatorname{dim}\left(V \backslash G_{1}\right)<k$ and hence $\operatorname{dim}(V \backslash G)<k$. Since also $\operatorname{dim}(G \backslash V) \leqslant \operatorname{dim}\left(G \backslash G_{1}\right)<k$, we obtain $\operatorname{dim}(G \triangle V)<k$. Now, by Lemma 2.5 , there is a definable set $S \subseteq G_{1}^{2}$ of dimension $<2 k$ and an $\mathcal{L}$-definable map $F: M^{2} \rightarrow M^{n}$ that agrees with · on $G_{1}^{2} \backslash S$. Let

$$
\Gamma=\left\{t \in G_{1}: \operatorname{dim}\left(S_{t}\right)<k\right\},
$$

and for $t \in \Gamma$,

$$
C_{t}=G_{1} \backslash S_{t} .
$$

Let also $C=\bigcup_{t \in \Gamma}\{t\} \times C_{t}$. By (D3), $\operatorname{dim}\left(G_{1} \backslash \Gamma\right)<k$, and $\operatorname{since} \operatorname{dim}\left(V \backslash G_{1}\right)<k, \operatorname{dim}(V \backslash$ $\Gamma)<k$. Similarly, $\operatorname{dim}\left(V \backslash C_{t}\right)<k$, for $t \in \Gamma$. It follows from (D3) that $\operatorname{dim}\left(V^{2} \backslash C\right)<2 k$. Finally, since $C \subseteq G_{1}^{2} \backslash S$, we have $F_{\lceil C}=\cdot \uparrow C$.

For the rest of this section, we fix $V, \Gamma, C_{t}, C$ and $F$ as above, and use their properties without any specific mentioning.

Note that since $\operatorname{dim}(G \backslash V)<k$, it follows that $\operatorname{dim}\left(G^{2} \backslash C\right)<2 k, \operatorname{dim}(G \backslash \Gamma)<k$, and, for $t \in \Gamma, \operatorname{dim}\left(G \backslash C_{t}\right)<k$.

\section{Step II: Group-like properties of $F$.}

Here we prove the existence of an $\mathcal{L}$-definable set $U \subseteq V^{2}$, with $\operatorname{dim}\left(V^{2} \backslash U\right)<2 k$, on which $F$ is continuous and behaves like a group operation, with $F(U) \subseteq V$. This is done through a series of lemmas.

Lemma 5.3. There is an $\mathcal{L}$-definable set $U \subseteq V^{2}$, with $\operatorname{dim}\left(V^{2} \backslash U\right)<2 k$, such that $F_{\uparrow U}$ is continuous and $F(U) \subseteq V$.

Proof. By o-minimality, there is an $\mathcal{L}$-definable set $U \subseteq V^{2}$, which is a finite union of $2 k$-cells, with $\operatorname{dim}\left(V^{2} \backslash U\right)<2 k$, such that $F_{\lceil U}$ is continuous. We claim that $F\left(C^{\prime}\right) \subseteq V$, for some set $C^{\prime} \subseteq V$ which is dense in $U$. Indeed, for every $t \in \Gamma$, consider the set

$$
C_{t}^{\prime}=\left\{x \in C_{t}: F(t, x) \in \Gamma\right\} .
$$

Since $F_{\uparrow C}=\cdot_{\uparrow C}$, we have that $\left.F(t,-)\right\rangle_{\uparrow C_{t}}$ is injective. Since also $\operatorname{dim}(G \backslash \Gamma)<k$, it follows from (D4) that $\operatorname{dim}\left(C_{t} \backslash C_{t}^{\prime}\right)<k$. Hence $\operatorname{dim}\left(V \backslash C_{t}^{\prime}\right)<k$. Moreover, $F\left(t, C_{t}^{\prime}\right) \subseteq \Gamma \subseteq V$. Let

$$
C^{\prime}=\bigcup_{t \in \Gamma}\{t\} \times C_{t}^{\prime}
$$


By (D3), $\operatorname{dim}\left(V^{2} \backslash C^{\prime}\right)<2 k$, and hence $\operatorname{dim}\left(U \backslash C^{\prime}\right)<2 k$. By Fact 2.1 , since $U$ is a finite union of $2 k$-cells, $C^{\prime}$ is dense in $U$. Moreover, $F\left(C^{\prime}\right) \subseteq V$, as required. Now, since $U \subseteq$ $\operatorname{cl}\left(C^{\prime}\right), V$ is closed and $F_{\lceil U}$ is continuous, it follows that $F(U) \subseteq V$.

Lemma 5.4. There is an $\mathcal{L}$-definable set $U \subseteq V^{2}$ with $\operatorname{dim}\left(V^{2} \backslash U\right)<2 k$, such that $F_{\lceil U}$ is injective in each coordinate.

Proof. It suffices to find an $\mathcal{L}$-definable set $U \subseteq V^{2}$ with $\operatorname{dim}\left(V^{2} \backslash U\right)<2 k$, such that $F_{\lceil U}$ is injective in the second coordinate. One can then similarly find $U^{\prime} \subseteq V^{2}$ with $\operatorname{dim}\left(V^{2} \backslash U^{\prime}\right)<2 k$ and $F_{\left\lceil U^{\prime}\right.}$ injective in the first coordinate, and the intersection $U \cap U^{\prime}$ is the desired set.

Suppose toward a contradiction that there is no such $U$. For every $t \in V$, let

$$
N(t)=\{x \in V: \exists y \in V, y \neq x \text { and } F(t, x)=F(t, y)\} .
$$

Then the set

$$
K=\{t \in V: \operatorname{dim} N(t)=k\}
$$

is $\mathcal{L}$-definable. So for any $t \in K, F(t,-)$ is not injective on any $\mathcal{L}$-definable subset of $V$ of co-dimension $<k$. So, by assumption and (D3), $\operatorname{dim} K=k$. Since $\Gamma$ is dense in $V$, there is $t \in K \cap \Gamma \neq \emptyset$. Since $t \in \Gamma, F(t,-){ }_{\uparrow}$ is injective. By Lemma 2.3, for $D=C_{t}$ and $X=V$, there is an $\mathcal{L}$-definable subset of $V$ of co-dimension $<k$ on which $F(t,-)$ is injective, contradicting $t \in K$.

Lemma 5.5. There is an $\mathcal{L}$-definable set $W \subseteq V^{3}$ with $\operatorname{dim}\left(V^{3} \backslash W\right)<3 k$, such that for every $(x, y, z) \in W$,

$$
F(x, F(y, z))=F(F(x, y), z) .
$$

Proof. By o-minimality, there is an $\mathcal{L}$-definable set $W \subseteq V^{3}$, which is a finite union of $3 k$-cells, with $\operatorname{dim}\left(V^{3} \backslash W\right)<3 k$, such that both maps $\bar{F}(-, F(-,-)): V^{3} \rightarrow M^{n}$ and $F(F(-,-),-): V^{3} \rightarrow M^{n}$ are continuous on $W$. It is thus enough to prove that $\left(^{*}\right)$ holds on a dense subset of $W$.

We observe that for every $(x, y, z) \in V^{3}$ with $x, y, F(x, y) \in \Gamma, F(y, z) \in C_{x}$ and $z \in$ $C_{y} \cap C_{F(x, y)}$, equation (*) holds, since both of its sides equal $x y z$. Hence, if, for every $x, y \in V$, we let

$$
Y_{x}=\Gamma \cap F(x,-)^{-1}(\Gamma)
$$

and

$$
Z_{x, y}=F(y,-)^{-1}\left(C_{x}\right) \cap C_{y} \cap C_{F(x, y)},
$$

then $(*)$ holds on the set

$$
T=\bigcup_{(x, y) \in \bigcup_{x \in \Gamma}\{x\} \times Y_{x}}\{(x, y)\} \times Z_{x, y} .
$$

Since $F_{\lceil C}={ }_{{ } C}$, $\operatorname{dim}(G \backslash \Gamma)<k$ and, for $x \in \Gamma, \operatorname{dim}\left(G \backslash C_{x}\right)<k$, it follows easily from (D4) that for every $x \in \Gamma$ and $y \in Y_{x}, \operatorname{dim}\left(G \backslash Y_{x}\right)<k$ and $\operatorname{dim}\left(G \backslash Z_{x y}\right)<k$. Since also $\operatorname{dim}(G \backslash \Gamma)<k$, (D3) implies that $\operatorname{dim}\left(G^{3} \backslash T\right)<3 k$, and hence $\operatorname{dim}\left(V^{3} \backslash T\right)<3 k$. Thus $\operatorname{dim}(W \backslash T)<3 k$, and by Fact $2.1, T$ is dense in $W$. 
We can refine the set $U$ in order to achieve two additional properties.

Corollary 5.6. Let $W$ be as in Lemma 5.5. Then, there is an $\mathcal{L}$-definable set $U \subseteq V^{2}$, such that

- $\operatorname{dim}\left(V^{2} \backslash U\right)<2 k$,

- $F_{\lceil U}$ is continuous, and injective in each coordinate,

- $F(U) \subseteq V$,

- for every $t \in V, \operatorname{dim}\left(V \backslash U_{t}\right)<k$,

- for every $(t, x) \in U, \operatorname{dim}\left(V \backslash W_{t, x}\right)<k$.

Proof. Let $U$ be as in Lemma 5.3. Define

$$
T=\left\{t \in V: \operatorname{dim}\left(V \backslash U_{t}\right)<k\right\}
$$

and

$$
U_{1}=\bigcup_{t \in T}\{t\} \times U_{t} .
$$

Since $\operatorname{dim}\left(V^{2} \backslash U\right)<2 k$, we obtain $\operatorname{dim}(V \backslash T)<k$ and $\operatorname{dim}\left(V^{2} \backslash U_{1}\right)<2 k$. Define also

$$
U_{2}=\left\{(t, x) \in V^{2}: \operatorname{dim}\left(V \backslash W_{t, x}\right)<k\right\} .
$$

Since $\operatorname{dim}\left(V^{3} \backslash W\right)<3 k$, we obtain $\operatorname{dim}\left(V^{2} \backslash U_{2}\right)<2 k$. The desired set $U$ is the intersection of $U_{1}, U_{2}$ and the set obtained in Lemma 5.4 .

For the rest of this section, we fix the sets $U$ and $W$ as above, and use their properties without any specific mentioning.

\section{Step III: Extracting an $\mathcal{L}$-definable set $X \subseteq V$ using $F$.}

In this step, we use $F$ to recover a suitable $\mathcal{L}$-definable set $X \subseteq V$ with $\operatorname{dim}(V \backslash X)<k$. The work from $\S 3$ plays an essential role here. The suitability of $X$ will be evident in Step IV.

Lemma 5.7. The set $X=F\left(\Gamma^{2} \cap U\right)$ is $\mathcal{L}$-definable with $\operatorname{dim}(V \backslash X)<k$.

Proof. By Corollary 3.8, $X$ is $\mathcal{L}$-definable, so we need to show that $\operatorname{dim}(V \backslash X)<k$. By cell decomposition, $V$ is a finite union of cells. Let $V^{\prime}$ be the union of all $2 k$-cells in this decomposition. We write $V$ for $V^{\prime}$. Since $X$ is $\mathcal{L}$-definable, it suffices to show that $X$ is dense in $V$. Pick any $t \in \Gamma$ and write $K=U_{t} \cap C_{t}$. So $\operatorname{dim}(\Gamma \backslash K)<k$. On the one hand, we have

$$
\Gamma \backslash t K \subseteq t(G \backslash K) \subseteq t(\Gamma \backslash K) \cup t(G \backslash \Gamma)
$$

and hence $\operatorname{dim}(\Gamma \backslash t K)<k$. Since $\operatorname{dim}(V \backslash \Gamma)<k$, we obtain $\operatorname{dim}(V \backslash t K)<k$. By Fact 2.1, $t K$ is dense in $V$. On the other hand,

$$
F(t, K)=t K .
$$

That is, $F\left(t, U_{t} \cap C_{t}\right)$ is dense in $V$, and hence so is $X$. 
Step IV: Constructing a definable embedding $h: X \rightarrow G$.

In this step, we embed $X$ into $G$, after proving the key property $\left(^{*}\right)$ from the introduction. For every $t \in X$ and $r \in G$, the set

$$
L_{t, r}=\{x \in V \cap G: F(t, x)=r x\}
$$

is definable.

Lemma 5.8. For every $s \in X$, there is unique $r \in G$, such that $\operatorname{dim}\left(G \backslash L_{s, r}\right)<k$.

Proof. Let $s=F(t, x)$, where $(t, x) \in \Gamma^{2} \cap U$. Recall that $\operatorname{dim}\left(V \backslash W_{t, x}\right)<k$. Let

$$
Y=W_{t, x} \cap F(x,-)^{-1}\left(C_{t}\right) \cap C_{x} .
$$

Then $\operatorname{dim}(G \backslash Y)<k$. Moreover, for every $y \in Y$, we have

$$
F(F(t, x), y)=F(t, F(x, y))=t F(x, y)=t x y .
$$

That is, for $r=t x$, we obtain $Y \subseteq L_{s, r}$. It follows that $\operatorname{dim}\left(G \backslash L_{s, r}\right)<k$.

The uniqueness of $r$ is clear, since otherwise we would obtain two sets $L_{s, r}$ and $L_{s, r^{\prime}}$ both contained in $G$ and having co-dimension $<k$ in $G$, a contradiction.

We now consider the map $h: X \rightarrow G$ given by

$$
\left.h(t)=r \Leftrightarrow \operatorname{dim}\left(G \backslash L_{t, r}\right)<k \text { (equivalently, } \operatorname{dim}\left(V \backslash L_{t, r}\right)<k\right) .
$$

Recall that for every $t \in X \subseteq V, \operatorname{dim}\left(V \backslash U_{t}\right)<k$.

Claim 5.9. $h$ is injective.

Proof. Suppose that for $t, s \in X$, we have $h(t)=h(s)=r$. Then $\operatorname{dim}\left(V \backslash L_{t, r}\right)<k$ and $\operatorname{dim}\left(V \backslash L_{t, s}\right)<k$. Therefore,

$$
U_{t} \cap U_{s} \cap L_{t, r} \cap L_{s, r} \neq \emptyset .
$$

For $x$ in that intersection, we have $F(t, x)=r x=F(s, x)$, and by injectivity of $F_{\lceil U}$ in the first coordinate, $t=s$.

Claim 5.10. For every $t \in \Gamma, \operatorname{dim}\left(G \backslash L_{t, t}\right)<k$. In particular, $h_{\lceil X \cap \Gamma}=i d$.

Proof. Let $t \in \Gamma$. Then for every $x \in C_{t}, F(t, x)=t x$. So, $C_{t} \subseteq L_{t, t}$. Since $\operatorname{dim}\left(G \backslash C_{t}\right)<$ $k$, the result follows.

Since $\operatorname{dim}(V \backslash X)<k$, we have $\operatorname{dim}(G \backslash X)<k$. Since also $\operatorname{dim}(G \backslash \Gamma)<k$, we obtain $\operatorname{dim}(G \backslash X \cap \Gamma)<k$. Therefore, by Claim 5.10, $\operatorname{dim}(G \backslash h(X))<k$.

\section{Step V: Concluding the proof of Theorem 5.1.}

It remains to show the following statement. 
Lemma 5.11. There is an $\mathcal{L}$-definable set $Z \subseteq X^{2}$ with $\operatorname{dim}\left(X^{2} \backslash Z\right)<2 k$ and $F(Z) \subseteq X$, such that for every $(t, x) \in Z$,

$$
h(F(t, x))=h(t) h(x) .
$$

Proof. We let

$$
Z=F^{-1}(X) \cap X^{2} \cap U .
$$

Clearly, $F(Z) \subseteq X$. We prove $\operatorname{dim}\left(X^{2} \backslash Z\right)<2 k$. Recall that $X \subseteq V$, and hence $\operatorname{dim}\left(X^{2} \backslash\right.$ $U)<2 k$. So, it suffices to prove that $\operatorname{dim}\left(X^{2} \backslash F^{-1}(X)\right)<2 k$. Let $t \in X \subseteq V$. Since $\operatorname{dim}\left(V \backslash\left(\Gamma \cap U_{t}\right)\right)<k$, we have $\operatorname{dim}\left(X \backslash\left(\Gamma \cap U_{t}\right)\right)<k$. Let $\Gamma^{\prime}=\Gamma \cap X$. Then for every $t \in \Gamma^{\prime}, \operatorname{dim}\left(X \backslash\left(\Gamma \cap U_{t}\right)\right)<k$, and

$$
F\left(t, \Gamma \cap U_{t}\right) \subseteq F\left(\Gamma^{2} \cap U\right)=X .
$$

Hence, the set

$$
\bigcup_{t \in \Gamma^{\prime}}\{t\} \times\left(\Gamma \cap U_{t}\right)
$$

belongs to $F^{-1}(X)$ and has co-dimension $<2 k$ in $X^{2}$, as required.

Now let $(t, x) \in Z$. For $y \in X$, denote $D_{y}=L_{y, h(y)}$. So $\operatorname{dim}\left(V \backslash D_{y}\right)<k$. By injectivity of $F_{\uparrow U}$ in the second coordinate, $F(x,-)^{-1}\left(D_{t}\right)$ has co-dimension $<k$ in $V$. Hence the set

$$
Y=D_{F(t, x)} \cap W_{t, x} \cap F(x,-)^{-1}\left(D_{t}\right) \cap D_{x}
$$

is non-empty. Take any $y \in Y \backslash\{1\}$. Then

$$
h(F(t, x)) y=F(F(t, x), y)=F(t, F(x, y))=h(t) F(x, y)=h(t) h(x) y,
$$

and hence $h(F(t, x))=h(t) h(x)$, as required.

This ends the proof of Theorem 5.1, and hence, by Theorem 4.4, also that of Theorem 1.1.

\section{Expansions by dense independent sets}

In this section, we let $\mathcal{M}=\langle M,<,+, \ldots\rangle$ be an o-minimal expansion of an ordered group, $P \subseteq M$ a dense dcl-independent set, and $\mathcal{N}=\langle\mathcal{M}, P\rangle$. We let dim be the large dimension coming from [17], as described in $\S 2.4$. Note that the assumption that $\mathcal{M}$ expands a group is not due to any reasons pertinent to the current work, but only because the accounts [8] and [17] that analyze this pair work under it.

Theorem 1.2 will follow from Theorem 1.1 and the following theorem.

Theorem 6.1. Every definable group is definably isomorphic to a strongly large group.

The rest of this section is devoted to proving Theorem 6.1. Since its statement is a first-order formula, we may assume that $\mathcal{N}$ is sufficiently saturated and make use of Fact 2.11. The proof of Theorem 6.1 is based on the cone decomposition theorem from [17]. The terminology of $\S 2.4$ applies here. A simplified formulation of the cone decomposition theorem is that every definable set $X \subseteq M^{n}$ is a small union of sets of 
the form $h(J)$, where $J$ is a supercone in some $M^{k}$, and $h: J \rightarrow M^{n}$ is an $\mathcal{L}$-definable continuous injective map. However, one can achieve some uniformity in the above decomposition, by stocking the different $J$ 's into finitely many families of supercones, each in a fixed $M^{k}$, and extending $h$ to every such family $\mathcal{L}$-definably and continuously. For $\mathcal{J}=\bigcup_{g \in S}\{g\} \times J_{g}$, we write $S=\pi(\mathcal{J})$.

Definition 6.2 (Cones). A set $C \subseteq M^{n}$ is a $k$-cone, $k \geqslant 0$, if there is a definable set $\mathcal{J}=$ $\bigcup_{g \in S}\{g\} \times J_{g}$, where $S \subseteq P^{m}$ and every $J_{g} \subseteq M^{k}$ is a supercone, and an $\mathcal{L}$-definable continuous map $h: V \subseteq M^{m+k} \rightarrow M^{n}$, where $V$ is cell, such that

(1) for every $g \in S, V_{g}=\operatorname{sh}\left(J_{g}\right)$,

(2) $C=h(\mathcal{J})$

(3) $h: \mathcal{J} \rightarrow M^{n}$ is injective.

A cone is a $k$-cone for some $k$.

Remark 6.3. It is important to note that if $C=h(\mathcal{J})$ is a cone as above, then for every $g \in S$, the set $h\left(g, J_{g}\right)$ is a full set (as in $\left.\S 2.3\right)$. Indeed, the map $h(g,-): \operatorname{sh}\left(J_{g}\right) \rightarrow M^{n}$ is $\mathcal{L}$-definable continuous and injective. By Fact $2.12, h\left(g, J_{g}\right)$ is a full set.

Fact 6.4 (Cone decomposition theorem). Every definable set is a finite disjoint union of cones.

Proof. This is a consequence of the cone decomposition theorem in [17] and subsequent work in [18]. A detailed proof is given in [16, Fact 4.7]. In that reference the universe of $\mathcal{M}$ is assumed to be $\mathbb{R}$, but this played no role in the particular proof.

We will need a further decomposition as follows.

Claim 6.5. Every $k$-cone is a finite disjoint union of $k$-cones $h(\mathcal{J})$, with $S=\pi(\mathcal{J})$, such that:

(1) every $g \in S$ has all its coordinates distinct,

(2) $S$ is either finite, or every coordinate projection of $S$ is infinite.

Proof. We first show that every $k$-cone $C \subseteq M^{n}$ can be written as a finite disjoint union of $k$-cones satisfying (1). Let $C=h(\mathcal{J})$, with $\mathcal{J}$ and $h: V \subseteq M^{m+k} \rightarrow M^{n}$, as in Definition 6.2. We work by induction on $m$. For $m=1$, the result obviously holds. Let $m>1$, and consider the set $T \subseteq S$ of all those elements whose at least two coordinates are the same. Without loss of generality, assume that for every $g \in T$, the first two coordinates are the same (otherwise the argument is similar). It is easy to see that $h\left(\bigcup_{g \in S \backslash T}\{g\} \times J_{g}\right)$ has the right form, and hence we may assume that $T=S$. Now, for $g \in T$, let $g^{\prime}$ denote the $(m-1)$-tuple obtained from $g$ by removing the first coordinate. Let

$$
T^{\prime}=\left\{g^{\prime} \in M^{m-1}: g \in T\right\}
$$

and

$$
V^{\prime}=\left(T^{\prime} \times M^{k}\right) \cap V
$$


and define $\mathcal{J}^{\prime}=\left\{J_{g^{\prime}}^{\prime}\right\}_{g^{\prime} \in T^{\prime}}$, where $J_{g^{\prime}}^{\prime}=J_{g}$, and $h^{\prime}: V^{\prime} \rightarrow M^{n}$ with $h^{\prime}\left(g^{\prime}, t\right)=h(g, t)$. Then $h(\mathcal{J})=h^{\prime}\left(\mathcal{J}^{\prime}\right)$, with $T^{\prime} \subseteq M^{m-1}$. By inductive hypothesis, the result follows.

Now, we show that every $k$-cone that satisfies (1) can be written as a finite disjoint union of sets satisfying $(1)$ and $(2)$. Let $C=h(\mathcal{J})$ be as above. We work again by induction on $m$. If $m=1$, the result obviously holds. Let $m>1$, and suppose that some coordinate projection of $S$ is not infinite, say the first, $\pi_{1}(S)=\left\{t_{1}, \ldots, t_{l}\right\}$. Then $\mathcal{J}$ is the finite disjoint union of $\mathcal{J}_{i}=\left\{J_{i g}\right\}_{g \in S_{t_{i}}}, i=1, \ldots, l$, where $J_{i g}=J_{\left(t_{i}, g\right)}$. Let $h_{i}: V_{t_{i}} \rightarrow M^{n}$ with $h_{i}(g, x)=h\left(t_{i}, g, x\right)$. Then each $h_{i}\left(\mathcal{J}_{i}\right)$ is still a $k$-cone satisfying $(1)$, and $S_{t_{i}} \subseteq M^{m-1}$. By inductive hypothesis, the result follows.

We will also need the following lemma.

Lemma 6.6. Every $k$-cone can be definably embedded into $M^{k+1}$.

Proof. Let $X=h(\mathcal{J}) \subseteq M^{n}$ be a $k$-cone, with $\mathcal{J}=\bigcup_{g \in S}\{g\} \times J_{g} \subseteq M^{m+k}$ and $h: V \rightarrow$ $M^{n}$ as in Definition 6.2. We first embed $S$ into $M$. By Fact 2.11, there are $\alpha_{1}, \ldots, \alpha_{m} \in M$ which are dcl-independent over $P$. Define $f: S \rightarrow M$ via

$$
\left(x_{1}, \ldots, x_{m}\right) \mapsto \alpha_{1} x_{1}+\cdots+\alpha_{2} x_{m} .
$$

By choice of $\alpha_{1}, \ldots, \alpha_{m}$, it follows that $f$ is injective. Now, since $h$ is injective, we can embed $X$ into $M^{k+1}$ via $F: h(g, t) \mapsto(f(g), t)$.

Corollary 6.7. Let $X$ be a definable set of dimension $k$. Then there are $n \in \mathbb{N}$ and a definable injective map $f: X \rightarrow M^{n}$ with $\operatorname{dim} c l(f(X))=k+1$.

Proof. By cone decomposition, $X$ is a finite union of cones. By Lemma 6.6, each of the cones can be definably embedded into $M^{k+1}$. Then $X$ can be definably embedded into finitely many disjoint copies of $M^{k+1}$, say, in $M^{k+2}$. The dimension of the closure of their union is still $k+1$.

Corollary 6.8. Let $X=Y \cup S$ be a definable set of dimension $k$, where $Y$ is a full set and $\operatorname{dim} S<k$. Then $X$ is in definable bijection with a strongly large set.

Proof. We may assume that $Y$ and $S$ are disjoint (after replacing $S$ by $S \backslash Y$ ). Since $\operatorname{dim} S<k$, applying Corollary 6.7 to $S$, we obtain $n$ and a definable injective map $f$ : $S \rightarrow M^{n}$ such that $\operatorname{dim} \operatorname{cl}(f(S)) \leqslant k$. On the other hand, since $X=Y \cup S$ has dimension $k$, we obtain $\operatorname{dim} Y=k$, and since $Y$ is strongly large, we get $\operatorname{dim} c l(Y)=k$. Fix any two distinct $a, b \in M$ and tuples $\alpha=(a, \ldots, a)$ and $\beta=(b, \ldots, b)$ such that the sets $Y^{\prime}:=\{\alpha\} \times Y$ and $S^{\prime}:=\{\beta\} \times f(S)$ belong to the same $M^{l}$. Then clearly $X$ is in definable bijection with $Y^{\prime} \cup S^{\prime}$, and $\operatorname{dim} Y^{\prime} \cup S^{\prime}=\operatorname{dim} \operatorname{cl}\left(Y^{\prime} \cup S^{\prime}\right)=k$, as needed.

Note that we only used that $Y$ is strongly large in the above proof.

Proof of Theorem 6.1. Let $G=\langle G, \cdot, 1\rangle$ be a definable group, with $G \subseteq M^{n}$ and $\operatorname{dim} G=k$. For $a, b \in G$, we write $a b$ for $a \cdot b$. By Fact 6.4 and Claim $6.5, G$ is a finite union of cones $C_{1}, \ldots, C_{p}$, each of the form $h(\mathcal{J})$, where $S=\pi(\mathcal{J})$ satisfies Claim 6.5 (1) \& (2). Suppose toward a contradiction that $G$ is not in definable bijection with any 
strongly large set. We claim that some $k$-cone $C$ among $C_{1}, \ldots, C_{p}$ must be of the form $h(\mathcal{J})$, where, in addition, $S=\pi(\mathcal{J})$ is infinite. Indeed, otherwise, $G$ would be the union of finitely many sets of the form $h\left(g, J_{g}\right)$ as in Remark 6.3, together with a set of dimension $<k$. The former sets are all full, and, by Lemma 2.8, their union is also full. Hence $G$ is a union of a full set and a set of dimension $<k$, contradicting Corollary 6.8.

Now fix a $k$-cone $C=h(\mathcal{J})$ among $C_{1}, \ldots, C_{p}$, with $S=\pi(\mathcal{J}) \subseteq P^{m}$ infinite and $m$ maximal such. By Claim 6.5(1) \& (2), we can find two distinct elements $g_{1}, g_{2} \in S$ with all their $2 m$ coordinates distinct. Let $\Sigma$ be the set of all those $2 m$ coordinates. Let also $A \subseteq M$ be a finite parameter set that is used to define all cones $C_{i}=h_{i}\left(\mathcal{J}_{i}\right)$ and their associated functions $h_{i}$ and families of supercones $\mathcal{J}_{i}$. Let $A_{0} \subseteq A$ be so that $A \subseteq \operatorname{dcl}\left(A_{0} P\right)$ and $A_{0}$ is dcl-independent over $P$.

Case: $k=0$. Since all of $C_{1}, \ldots, C_{p}$ are 0 -cones, we may write $C_{i}=h_{i}\left(S_{i}\right)$, where $S_{i} \subseteq P^{k_{i}}$, for some $l_{i} \leqslant m$. Let $i$ and $g_{3} \in S_{i}$ be so that

$$
h\left(g_{1}\right) h\left(g_{2}\right)=h_{i}\left(g_{3}\right) .
$$

Since $|\Sigma|=2 m$, there must be $a \in \Sigma \backslash g_{3}$. Say $a \in g_{2} \backslash g_{1}$ (if $a \in g_{1} \backslash g_{2}$, the argument is symmetric). By injectivity of $h, a \in \operatorname{dcl}\left(g_{1}, g_{3}, A_{0}, P_{0}\right)$, contradicting the fact that $A_{0}$ is dcl-independent over $P$.

Case: $k>0$. We need the following claim.

Claim. There are a $k$-cone $D=h^{\prime}\left(\mathcal{J}^{\prime}\right)$ among the $C_{i}$ 's, with $\mathcal{J}^{\prime}=\bigcup_{g \in S^{\prime}}\{g\} \times J_{g}^{\prime}$, a tuple $g_{3} \in S^{\prime}$, and a triple $\left(t_{1}, t_{2}, t_{3}\right) \in J_{g_{1}} \times J_{g_{2}} \times J_{g_{3}}$, such that $t_{1} t_{3} A_{0} P$ is dcl-independent, and

$$
\text { (*) } \quad h\left(g_{1}, t_{1}\right) h\left(g_{2}, t_{2}\right)=h^{\prime}\left(g_{3}, t_{3}\right) .
$$

Proof of the claim. Let $X=J_{g_{1}} \times J_{g_{2}}$. Then $X$ is a supercone in $M^{2 k}$. By (D6), there is a definable set $Z \subseteq X$ with $\operatorname{dim} Z<2 k$ and an $\mathcal{L}$-definable map $F: M^{2 k} \rightarrow M^{n}$ such that the map

$$
\left(t_{1}, t_{2}\right) \mapsto h\left(g_{1}, t_{1}\right) h\left(g_{2}, t_{2}\right)
$$

agrees with $F$ on $X^{\prime}:=\left(J_{g_{1}} \times J_{g_{2}}\right) \backslash Z$. By o-minimality, there is an open cell $U \subseteq \operatorname{cl}(X)$, such that $F_{\lceil U}$ is continuous. By [17, Lemma 4.16], $U \cap X$ is a supercone in $M^{2 k}$, and hence the set $T=(U \cap X) \backslash Z$ also has dimension $2 k$. By Fact 2.11 , there is $\left(t_{1}, t_{2}\right) \in T$, which is dcl-independent over $A_{0} P$. Moreover, by [17, Lemma 5.10], there are a $k^{\prime}$-cone $D$ among the $C_{i}$ 's, say $D=h^{\prime}\left(\mathcal{J}^{\prime}\right)$, with $k^{\prime} \leqslant k$ and $\mathcal{J}^{\prime}=\bigcup_{g \in S^{\prime}}\{g\} \times J_{g}^{\prime}$, and $g_{3} \in S^{\prime}$ such that $F(T) \subseteq h^{\prime}\left(g_{3}, J_{g_{3}}^{\prime}\right)$. Let $t_{3} \in J_{g_{3}}^{\prime} \subseteq M^{k^{\prime}}$ be so that

$$
h\left(g_{1}, t_{1}\right) h\left(g_{2}, t_{2}\right)=h^{\prime}\left(g_{3}, t_{3}\right) .
$$

Since $t_{2} \in \operatorname{dcl}\left(t_{1}, t_{3}, A_{0}, P\right)$, it follows that $\left(t_{1}, t_{3}\right)$ is also dcl-independent over $A_{0} P$ and has dimension at least $2 k$. Hence $k^{\prime}=k$.

Let $D$ and $g_{3}$ be as in the claim. By maximality of $m$, it must be that $g_{3} \in S^{\prime} \subseteq P^{l}$, for some $l \leqslant m$. Since all $2 m$ coordinates of $g_{1}, g_{2}$ are distinct, there must be $a \in \Sigma \backslash g_{3}$. Say $a \in g_{2} \backslash g_{1}$. By $(*)$,

$$
h\left(g_{2}, t_{2}\right)=h\left(g_{1}, t_{1}\right)^{-1} h^{\prime}\left(g_{3}, t_{3}\right),
$$


and hence

$$
a \in g_{2} \subseteq \operatorname{dcl}\left(g_{1}, g_{3}, t_{1}, t_{3}, A_{0}, P_{0}\right)
$$

contradicting the fact that $t_{1} t_{2} A_{0}$ is dcl-independent over $P$.

Acknowledgements. I wish to thank Ya'acov Peterzil for pointing out the relevant literature and discussing the proof of the group chunk theorem in $\S 4$. The relevant discussions took place during the trimester in model theory, combinatorics and valued fields, 2018, at the Institut Henri Poincaré. I also thank Alfred Dolich and Philipp Hieronymi for suggesting that there should be no new definable groups in the setting of Theorem 1.2. Finally, I thank Chris Miller for his helpful feedback on an earlier version of this paper, and the referee for many valuable comments that helped to improve its presentation.

\section{References}

1. E. Baro And A. Martin-Pizarro, Small groups in dense pairs, Preprint, 2018, arXiv: $1801.08744 \mathrm{v} 1$.

2. O. Belegradek And B. Zilber, The model theory of the field of reals with a subgroup of the unit circle, J. Lond. Math. Soc. (2) 78 (2008), 563-579.

3. A. Berenstein, C. Ealy And A. Günaydin, Thorn independence in the field of real numbers with a small multiplicative group, Ann. Pure Appl. Logic 150 (2007), 1-18.

4. A. Berenstein And E. Vassiliev, Geometric structures with a dense independent subset, Selecta Math. (N.S.) 22 (2016), 191-225.

5. A. Berenstein And E. Vassiliev, Fields with a dense-codense linearly independent multiplicative subgroup, Preprint, 2017, Modnet 1238. Archive for Mathematical Logic, to appear.

6. G. Boxall and P. Hieronymi, Expansions which introduce no new open sets, J. Symbolic Logic 77(1) (2012), 111-121.

7. A. Dolich, C. Miller And C. Steinhorn, Structures having o-minimal open core, Trans. Amer. Math. Soc. 362 (2010), 1371-1411.

8. A. Dolich, C. Miller and C. Steinhorn, Expansions of o-minimal structures by dense independent sets, Ann. Pure Appl. Logic 167 (2016), 684-706.

9. L. VAN DEN DRIES, Remarks on Tarski's problem concerning $(R,+,-$, exp $)$, in Logic Colloquium (1982) (ed. G. Lolli, G. Longo And A. MarcJa), pp. 97-121 (North-Holland, 1984).

10. L. VAN DEN DrIEs, Dense pairs of o-minimal structures, Fund. Math. 157 (1988), 61-78.

11. L. VAN DEN DRIES, Dimension of definable sets, algebraic boundedness and henselian fields, Ann. Pure Appl. Logic 45 (1989), 189-209.

12. L. VAN DEN DRIES, Weil's group chunk theorem: A topological setting, Illinois J. Math. 34 (1990), 127-139.

13. L. VAN DEN Dries, Tame Topology and O-minimal Structures (Cambridge University Press, Cambridge, 1998).

14. L. van Den Dries And A. Günaydin, The fields of real and complex numbers with a small multiplicative group, Proc. Lond. Math. Soc. 93 (2006), 43-81.

15. P. Eleftheriou, Non-standard lattices and o-minimal groups, Bull. Symbolic Logic 19 (2013), 56-76. 
16. P. Eleftheriou, Counting algebraic points in expansions of o-minimal structures by a dense set, Preprint, 2018, arXiv:1708.03936v3.

17. P. Eleftheriou, A. Günaydin And P. Hieronymi, Structure theorems in tame expansions of o-minimal structures by dense sets, Preprint, 2017, arXiv:1510.03210v4.

18. P. Eleftheriou, A. Günaydin and P. Hieronymi, The Choice Property in tame expansions of o-minimal structures, Preprint, 2017, arXiv:1708.03896v1.

19. P. Eleftheriou And Y. Peterzil, Definable groups as homomorphic images of semilinear and field-definable groups, Selecta Math. (N.S.) 18 (2012), 905-940.

20. P. Eleftheriou, Y. Peterzil and J. Ramakrishnan, Interpretable groups are definable, J. Math. Log. 14 (2014), 1450002.

21. A. Fornasiero, Groups and rings definable in d-minimal structures, Preprint, 2012, arXiv:1205.4177v1.

22. A. GüNAydin And P. Hieronymi, The real field with the rational points of an elliptic curve, Fund. Math. 215 (2011), 167-175.

23. E. Hrushovski, The Mordell-Lang conjecture for function fields, J. Amer. Math. Soc. 9 (1996), 667-690.

24. E. Hrushovski, Y. Peterzil and A. Pillay, Groups, measures, and the NIP, J. Amer. Math. Soc. 21 (2008), 563-596.

25. J. Johns, An Open Mapping Theorem for O-Minimal Structures, J. Symbolic Logic 66 (2001), 1817-1820.

26. J. Knight, A. Pillay and C. Steinhorn, Definable sets in ordered structures II, Trans. Amer. Math. Soc. 295 (1986), 593-605.

27. C. Miller And P. Speissegger, Expansions of the real line by open sets: o-minimality and open cores, Fund. Math. 162 (1999), 193-208.

28. C. Miller And S. Starchenko, A growth dichotomy for o-minimal expansions of ordered groups, Trans. Amer. Math. Soc. 350(9) 3505-3521.

29. Y. Peterzil, Returning to semi-bounded sets, J. Symbolic Logic 74 (2009), 597-617.

30. J. Pila, O-minimality and the André-Oort conjecture for $\mathbb{C}^{n}$, Ann. Math. 173 (2011), $1779-1840$

31. J. Pila And A. J. Wilkie, The rational points of a definable set, Duke Math. J. 133 (2006), 591-616.

32. A. Pillay and C. Steinhorn, Definable sets in ordered structures I, Trans. Amer. Math. Soc. 295 (1986), 565-592.

33. A. Robinson, Solution of a problem of Tarski, Fund. Math. 47 (1959), 79-204.

34. R. Wencel, Groups, group actions and fields definable in first-order topological structures, Math. Log. Quart. 58 (2012), 449-467. 